SPIN

Vol. 4, No. 4 (2014) 1440025 (8 pages)

(C) The Authors

DOI: $10.1142 /$ S2010324714400256

\title{
EPITAXIAL GROWTH OF CUBIC MnSb ON GaAs AND InGaAs(111)
}

\author{
GAVIN R. BELL ${ }^{* *}$, CHRISTOPHER W. BURROWS ${ }^{*}$, , THOMAS P. A. HASE* \\ MARK J. ASHWIN*,", SEAN R. C. MCMITCHELL*,**, \\ ANA M. SANCHEZ ${ }^{* \dagger \dagger}$ and JAMES D. ALDOUS ${ }^{\dagger,+*}$ \\ *Department of Physics, University of Warwick \\ Coventry $\mathrm{CV}_{4}$ 7AL, UK \\ ${ }^{\dagger}$ London Centre for Nanotechnology \\ University College London \\ 17-19 Gordon Street London WC1H OAH, UK \\ *gavin.bell@warwick.ac.uk \\ §c.burrows@warwick.ac.uk \\ Tt.p.a.hase@warwick.ac.uk \\ ${ }$ m.j.ashwin@warwick.ac.uk \\ **s.mcmitchell@warwick.ac.uk \\ †a.m.sanchez@warwick.ac.uk \\ \$j.aldous@ucl.ac.uk
}

Received 30 April 2014

Accepted 1 September 2014

Published 16 October 2014

\begin{abstract}
The cubic polymorph of the binary transition metal pnictide (TMP) MnSb, c-MnSb, has been predicted to be a robust half-metallic ferromagnetic (HMF) material with minority spin gap $\gtrsim 1 \mathrm{eV}$. Here, MnSb epilayers are grown by molecular beam epitaxy (MBE) on GaAs and $\mathrm{In}_{0.5} \mathrm{Ga}_{0.5} \mathrm{As}(111)$ substrates and analyzed using synchrotron radiation X-ray diffraction. We find polymorphic growth of $\mathrm{MnSb}$ on both substrates, where c-MnSb co-exists with the ordinary niccolite $\mathrm{n}-\mathrm{MnSb}$ polymorph. The grain size of the c-MnSb is of the order of tens of nanometer on both substrates and its appearance during MBE growth is independent of the very different epitaxial strain from the GaAs $(3.1 \%)$ and $\mathrm{In}_{0.5} \mathrm{Ga}_{0.5} \mathrm{As}(0.31 \%)$ substrates.
\end{abstract}

Keywords: MnSb; half-metallic ferromagnetism; polymorphism; epitaxy; X-ray diffraction.

\section{Introduction}

Half-metallic ferromagnetism ${ }^{1,2}$ is a highly desirable property for advanced spintronic devices which require very high spin polarization at the Fermi level and has been explored in several classes of materials. ${ }^{3,4}$ The cubic polymorphs of many binary transition metal pnictides (TMPs), such as MnAs or CrSb, have been predicted to be half-metallic ferromagnetic

$\$$ Corresponding author.

This is an Open Access article published by World Scientific Publishing Company. It is distributed under the terms of the Creative Commons Attribution 3.0 (CC-BY) License. Further distribution of this work is permitted, provided the original work is properly cited. 
(HMF) materials with Curie temperatures $\left(T_{C}\right)$ well above room temperature which is essential if they are to be exploited in any functional device. ${ }^{5-7}$ Many TMPs normally adopt a double hexagonal closepacked $\mathrm{B} 88_{1}$ niccolite structure (abbreviated $\mathrm{n}$-), which has $\mathrm{ABAC}$ stacking along the $c$-axis $(\mathrm{A}=$ transition metal, $\mathrm{B}, \mathrm{C}=$ pnictogen). The structure of the cubic (c-) TMP polymorphs are predicted to be significantly less energetically favorable than the n-polymorphs under normal growth conditions. However, because the TMPs have excellent engineering compatibility with many mainstream III-V and Group IV semiconductor materials, ${ }^{\mathrm{a}}$ their cubic polymorphs in particular have enormous potential as highly spinpolarized contacts for hybrid semiconductor spintronics. Combining TMPs with semiconductors allows the greatest possible flexibility in spintronic device design: arbitrary all-epitaxial ferromagnet/antiferromagnet/semiconductor heterostructures could be grown (e.g., $\mathrm{MnSb} / \mathrm{CrSb} / \mathrm{In}_{1-x} \mathrm{Ga}_{x} \mathrm{As}$ ).

While most band structure calculations made using density functional theory (DFT) apply to zero temperature it has been known for some years that the nonzero temperature behavior of HMFs is very important in determining their real-world spin polarization and hence device performance. ${ }^{8-10}$ The possibility of a critical temperature $T^{*} \ll T_{C}$ for the onset of a reduction in the spin polarization was highlighted by a recent DFT calculation employing the disordered local moments (DLM) approach to model nonzero temperature band structures. ${ }^{5}$ In that work, both NiMnSb (the canonical HMF semiHeusler alloy, ${ }^{2}$ more consistently labeled $\mathrm{MnNiSb}^{11}$ ) and c-MnSb were studied. NiMnSb has a small minority spin gap $0.5 \mathrm{eV}$ giving a low $T^{*}$ in the region of $100 \mathrm{~K}$. This low value of $T^{*}$ arises from defect-like states in the minority spin gap, whose spectral weight increases with spin disorder, and easily reaches the Fermi level with an overall magnetization reduction of only $5 \%$. Conversely the large minority spin gap $\geq 1 \mathrm{eV}$ of $\mathrm{c}-\mathrm{MnSb}$, together with a mid-gap Fermi level, means that magnetization reduction must reach around $20 \%$ before minority spin magnetic disorder states reach the Fermi level, giving a $T^{*}$ probably in excess of $300 \mathrm{~K} .{ }^{5}$ The prospect for room-temperature $\mathrm{HMF}$ behavior in $\mathrm{c}-\mathrm{MnSb}$ makes it a highly promising spintronic material.
Several groups have investigated the growth of cubic TMP polymorphs by molecular beam epitaxy (MBE) on substrates with square symmetry. $6,12,13$ The presence of cubic phases has been inferred from structural measurements such as X-ray diffraction (XRD) and transmission electron microscopy (TEM), while for some ultra-thin films $(<1 \mathrm{~nm})$ normally antiferromagnetic materials such as CrAs can show slightly open hysteresis loops, suggesting the presence of a ferromagnetic cubic polymorph. ${ }^{14}$ However, detailed DFT work suggested that these ultra-thin films were not actually cubic in structure and the observed magnetic hysteresis was likely due to uncompensated spins in a highly strained distorted orthorhombic epilayer. Furthermore, for such ultra-thin films, interpretation of TEM and XRD data is far from being unambiguous. More recently it has been claimed that c-MnAs can be grown directly on $\operatorname{InP}(001)$ alongside the ordinary hexagonal phase. ${ }^{15,16}$ We recently demonstrated the growth of c-MnSb within n-MnSb films on $\operatorname{GaAs}(111)$, where the large grain sizes of the $\mathrm{c}-\mathrm{MnSb}(\geq 10 \mathrm{~nm})$ made structural identification more straightforward.

In this work we extend our MBE growth of $\mathrm{MnSb}$ from $\mathrm{InP},{ }^{17} \mathrm{GaAs}^{18}$ and $\mathrm{Ge}^{19}$ to relaxed $\mathrm{In}_{0.5} \mathrm{Ga}_{0.5} \mathrm{As}$ (111) virtual substrates. Furthermore, $\operatorname{In}_{1-x} \mathrm{Ga}_{x} \mathrm{As}$ structures are attractive for semiconductor spintronic applications thanks to their high electron mobility, high Landé $g$-factor and low Schottky barriers. ${ }^{20}$ Furthermore, the nominal lattice mismatch to $\mathrm{n}-\mathrm{MnSb}$ is only $0.31 \%$ for $\mathrm{In}_{0.5} \mathrm{Ga}_{0.5} \mathrm{As}$, compared to $3.1 \%$ for GaAs. Hence, by comparing the growth of $\mathrm{MnSb}$ grown directly on GaAs with that on $\operatorname{In}_{1-x} \mathrm{Ga}_{x} \mathrm{As}$, it is possible to determine whether the substrate lattice strain plays a role in polymorph formation. In fact, we will show that the formation of c-MnSb occurs in a very similar fashion on both GaAs and $\operatorname{In}_{0.5} \mathrm{Ga}_{0.5}$ As substrates suggesting that the substrate strain is of minimal importance, at least in this family of substrate materials. These results are consistent with our earlier suggestion that the c-MnSb forms epitaxially on top of $\mathrm{n}-\mathrm{MnSb}$ in a mixed c-MnSb/n-MnSb polymorphic layer, rather than in contact with the substrate. The outlook for exploiting TMP polymorphs in spintronics is briefly discussed.

aBy "engineering compatibilty" we mean straightforward epitaxial growth, usually favorable interface chemistry, and ease of device processing and fabrication. 


\section{Experimental Details}

All MnSb films were grown by MBE in a dedicated home-built chamber, using wafer pieces cut to typically $8 \times 8 \mathrm{~mm}$. After sonication with organic solvents and rinsing with deionized water to remove dust and cutting debris, substrate wafers were cleaned in vacuo by careful degassing, gentle Ar ion sputtering ( $500 \mathrm{eV}, 0.5 \mu \mathrm{A}, 10 \mathrm{~min})$ and annealing without incident Group V flux. For the (111)A oriented substrates this produced the expected $(2 \times 2)$ reconstructions on both GaAs and $\mathrm{In}_{0.5} \mathrm{Ga}_{0.5}$ As. $\mathrm{Mn}$ and $\mathrm{Sb}$ effusion cells operating close to $857^{\circ} \mathrm{C}$ and $355^{\circ} \mathrm{C}$, respectively produced an $\mathrm{Sb}_{4}: \mathrm{Mn}$ beam equivalent pressure ratio of $6.6: 1$ as measured by a retractable ionization gauge. This appears to be the optimum flux ratio for $\mathrm{MnSb}$ polymorph formation on $\mathrm{GaAs}(111)$ and the fluxes were balanced carefully before each growth. Sample rotation during growth is not possible within our MBE system. The growth rate was $2.7 \mathrm{~nm} \mathrm{~min}^{-1}$ and the substrate temperature was maintained at $(415 \pm 5)^{\circ}$ as measured by a thermocouple on the sample manipulator. Films varying in thickness from $1 \mathrm{~nm}$ to $300 \mathrm{~nm}$ were grown: here we focus on $\mathrm{MnSb}$ films in the thickness range $100 \mathrm{~nm}$ to $300 \mathrm{~nm}$ where cubic polymorphs can be found.

Both GaAs wafers (moderately n-doped, exactly oriented) and $400 \mathrm{~nm}$ thick $\operatorname{In}_{0.5} \mathrm{Ga}_{0.5}$ As virtual substrates were used. The latter were grown in a separate Varian MBE system on $50 \mathrm{~mm}$ (2 inch) $\operatorname{GaAs}(111) \mathrm{A}$ wafers. $\mathrm{In}_{0.5} \mathrm{Ga}_{0.5}$ As was deposited under standard conditions on to a $100 \mathrm{~nm}$ GaAs buffer layer (InGaAs growth rate $16.7 \mathrm{~nm} \mathrm{~min}^{-1}$, substrate temperature $500^{\circ} \mathrm{C}$, sample rotation) and a protective As cap was deposited after epilayer completion. Due to the high lattice strain, the surface roughness and crystalline mosaic were significantly higher for the $\operatorname{In}_{0.5} \mathrm{Ga}_{0.5}$ As virtual substrates than for the GaAs wafers. The virtual substrates were transferred through air, cut and mounted in the same way as GaAs wafers and prepared for subsequent $\mathrm{MnSb}$ deposition using the same protocols except for a longer pre-anneal to desorb the As cap.

High-resolution XRD experiments were performed both in-house and at three synchrotron radiation facilities ${ }^{\mathrm{b}}$ all equipped with multi-circle diffractometers. In this work, we show synchrotron XRD data only, all of which were obtained with samples at room temperature under flowing dry nitrogen using $10 \mathrm{keV}$ photons monochromated using $\mathrm{Si}(111)$ crystals. Angular data, which were recorded in a triple-axis geometry using suitable analyzer crystals, have been reduced to reciprocal lattice units in which the $Q_{z}$ direction is defined as normal to the substrate surface and hence parallel to a [111] direction. Scans were recorded as a function of both $Q_{z}$ and the orthogonal, in-plane, $Q_{x}$, directions. Reciprocal space maps (RSMs) were obtained in symmetric (out-of-plane) and asymmetric (in-plane) diffraction geometries. The TEM experiments were performed using Jeol ARM-200F and 2100 microscopes operating at $200 \mathrm{keV}$.

\section{Results and Discussion}

We present TEM images from typical $\mathrm{MnSb} /$ $\mathrm{In}_{0.5} \mathrm{Ga}_{0.5} \mathrm{As} / \mathrm{GaAs}(111) \mathrm{A}$ heterostructures and then compare XRD data from $\mathrm{MnSb} / \mathrm{In}_{0.5} \mathrm{Ga}_{0.5} \mathrm{As}$ and $\mathrm{MnSb} / \mathrm{GaAs}$. In Fig. 1, are shown two brightfield TEM images from a $300 \mathrm{~nm}$ thick MnSb film on $\mathrm{In}_{0.5} \mathrm{Ga}_{0.5} \mathrm{As}(111) \mathrm{A}$. The overview of the heterostructure is shown in (a) and the arrows highlight the $\mathrm{GaAs}^{-} \mathrm{In}_{0.5} \mathrm{Ga}_{0.5} \mathrm{As}$ interface (bottom) and $\mathrm{In}_{0.5} \mathrm{Ga}_{0.5}$ As-MnSb interface (top). The $\mathrm{In}_{0.5} \mathrm{Ga}_{0.5} \mathrm{As}$ layer is highly defective with many threading dislocations, as expected from the large lattice mismatch $(3.7 \%)$ with the substrate. However, its surface is reasonably smooth and has a sharp interface with the $\mathrm{MnSb}$ overlayer. Since the $\mathrm{MnSb}$ is closely matched to the relaxed $\operatorname{In}_{0.5} \mathrm{Ga}_{0.5}$ As virtual substrate, the density of dislocations is much lower in the MnSb film. However, non-niccolite structures can be discerned in the MnSb films. In particular, the surface region of this film shows a granular morphology with different crystal structures. This layer is visible in Fig. 1(b) where the arrow highlights the interface between a granular surface layer incorporating non-B $8{ }_{1}$ structures and the underlying "pure" n-MnSb.

This surface layer is shown in more detail in Fig. 2(a). The crystallite size within this layer is typically around $25 \mathrm{~nm}$ both laterally and in terms of overall layer thickness. A high-resolution TEM image obtained on a single crystallite from within this surface layer is shown in Fig. 2(b). The atomic columns are readily resolved and their symmetry is

bBeamline X22C at NSLS (Brookhaven National Laboratory, USA), the XMaS facility at the ESRF (Grenoble, France) and beamline 16 at Diamond Light Source, UK. 


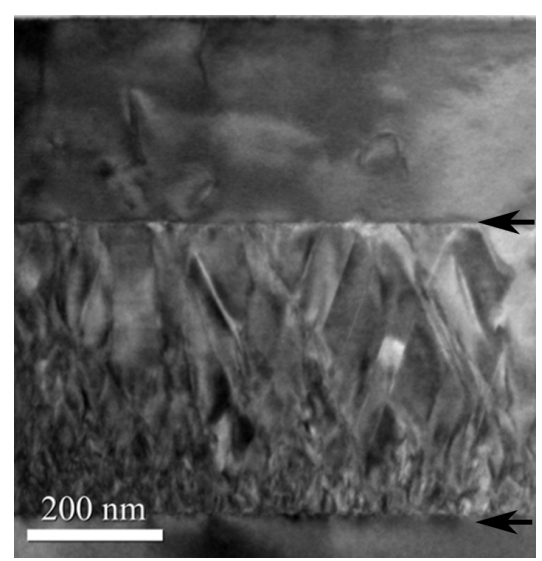

(a)

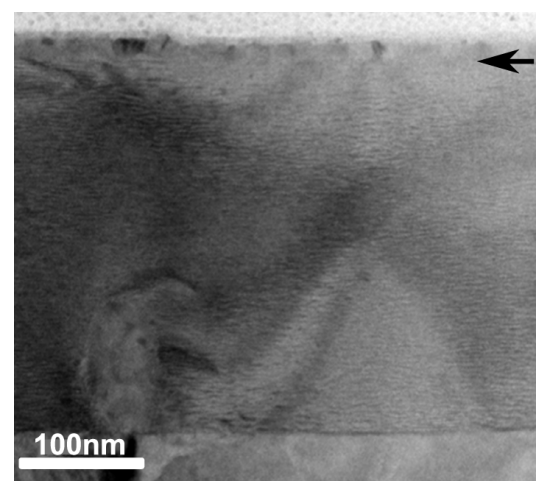

(b)

Fig. 1. Bright-field TEM images of a typical MnSb/ $\mathrm{In}_{0.5} \mathrm{Ga}_{0.5} \mathrm{As} / \mathrm{GaAs}$ heterostructure. Black arrows highlight the principal interfaces: in (a) between GaAs and $\operatorname{In}_{0.5} \mathrm{Ga}_{0.5} \mathrm{As}$ (bottom) and between $\operatorname{~n}_{0.5} \mathrm{Ga}_{0.5} \mathrm{As}$ and $\mathrm{MnSb}$ (top), and in (b) between the two polymorphs of $\mathrm{MnSb}$.

consistent with a cubic structure oriented with (111) planes parallel to the $\mathrm{n}-\mathrm{MnSb}(0001)$ interface. A Fourier transform of the image is shown in the inset of Fig. 2(b). This part of the MnSb film comprises both c-MnSb grains and a continuation of the $\mathrm{n}-\mathrm{MnSb}$ structure, i.e., it is not a simple epitaxial layer of c-MnSb on top of $\mathrm{n}-\mathrm{MnSb}$. The appearance of this granular structure is strikingly similar to c$\mathrm{MnSb}$ on $\mathrm{n}-\mathrm{MnSb}$ grown directly on $\operatorname{GaAs}(111) .{ }^{5}$

In Fig. 3, are shown selected synchrotron XRD data for a typical $\mathrm{MnSb} / \mathrm{In}_{0.5} \mathrm{Ga}_{0.5} \mathrm{As} / \mathrm{GaAs}$ heterostructure. Sharp line features on the symmetric diffractograms in (a) and (b) are due to multiple scattering from the substrate. The expected substrate and virtual substrate peaks are present along with n-MnSb with its $c$-axis out-of-plane. Panel (b) shows a smaller angular range around the lowest order peaks. The sharpest and most intense peak is

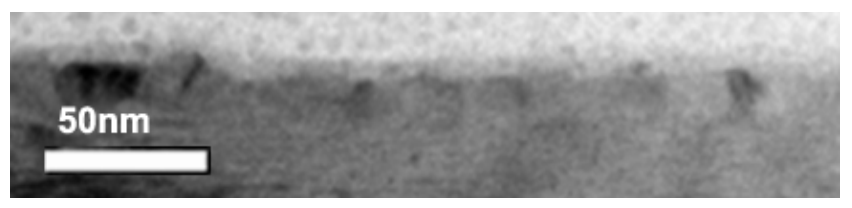

(a)

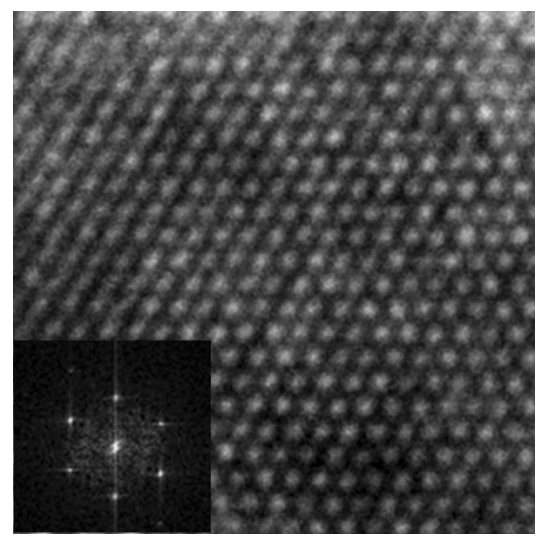

(b)

Fig. 2. Bright-field (a) and high-resolution (b) TEM images from a typical $\mathrm{MnSb} / \mathrm{In}_{0.5} \mathrm{Ga}_{0.5} \mathrm{As} / \mathrm{GaAs}$ heterostructure. Image (a) highlights the grain size of around $25 \mathrm{~nm}$ in the nonniccolite structured surface layer, while (b) was obtained from one of these grains and shows symmetry consistent with c-MnSb (111). A Fourier transform of image (b) is inset.

due to the $\operatorname{GaAs}(111)$ substrate (the peak is within $10^{-3} \AA^{-1}$ of the position expected for GaAs at room temperature). The virtual substrate appears at lower $Q_{z}$ due to its larger lattice parameter, and the peak is slightly broadened due to residual strain near the GaAs interface. The $\mathrm{n}-\mathrm{MnSb}(0002)$ peak is intense and quite symmetric reflecting the high crystalline quality of the epilayer. Lattice parameters derived from fitting these peaks are $a=(5.853 \pm$ $0.001) \AA$ and $c=(5.768 \pm 0.001) \AA$ for $\operatorname{In}_{0.5} \mathrm{Ga}_{0.5} \mathrm{As}$ and $\mathrm{n}-\mathrm{MnSb}$, respectively. The value for the $c$-lattice parameter of $\mathrm{n}-\mathrm{MnSb}$ is consistent with that found in films grown on other substrates ${ }^{5,19}$ as well as the bulk material. ${ }^{21}$

Figure 3(c) shows a RSM about the GaAs(422) reflection (asymmetric diffraction geometry). Several additional diffraction features appear due to the virtual substrate and the two polymorphs of $\mathrm{MnSb}$. The $n-M n S b(1 \overline{1} 05)$ and $(1 \overline{1} 04)$ peaks are present in the correct ratio derived when compared with intensities derived from the structure factors of the ideal niccolite structure. The presence of both c$\mathrm{MnSb}$ (422) and (133) reflections is interesting. For a single epitaxial orientation of c-MnSb on $\mathrm{n}-\mathrm{MnSb}$, 


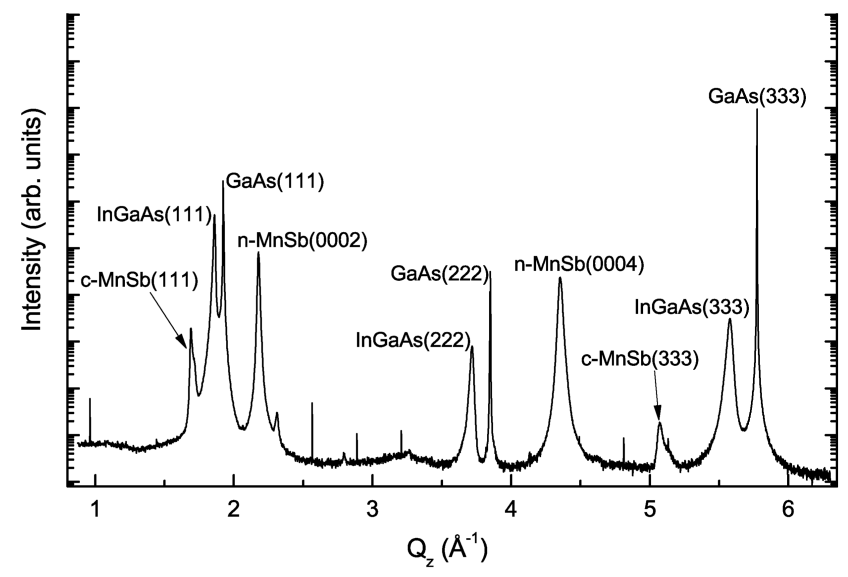

(a)

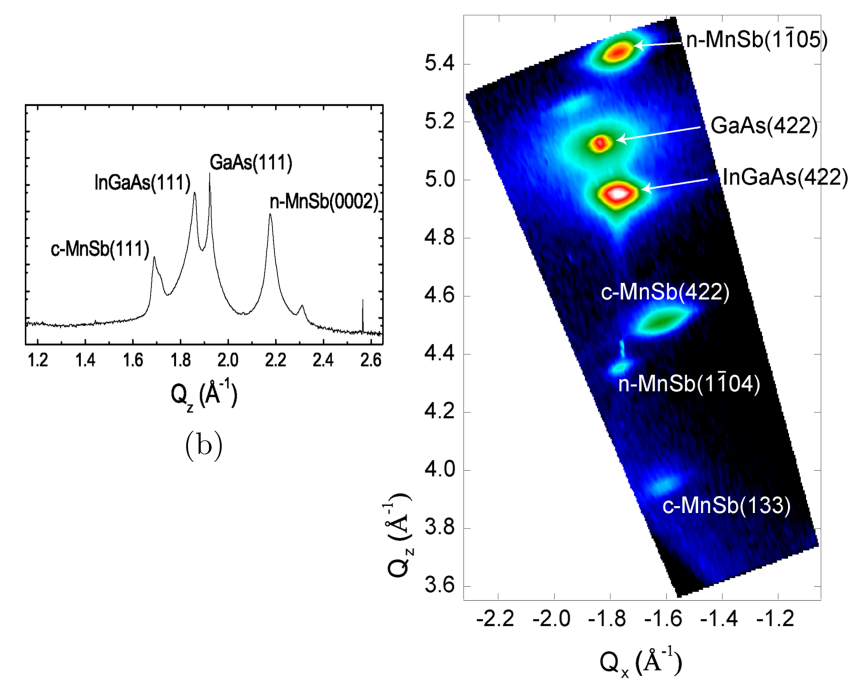

(c)

Fig. 3. Synchrotron XRD data for a typical MnSb/ $\mathrm{In}_{0.5} \mathrm{Ga}_{0.5} \mathrm{As} / \mathrm{GaAs}$ heterostructure. Panel (a) shows a standard out-of-plane (symmetric) diffractogram. All the main peaks are labeled and correspond to the expected $\mathrm{GaAs}$ and $\mathrm{In}_{0.5} \mathrm{Ga}_{0.5} \mathrm{As}$ (111)-type reflections and $\mathrm{n}-\mathrm{MnSb}(0002)$ reflections. A clear set of additional weaker peaks is assigned to c-MnSb. Panel (b) shows a more detailed view of the group of lowest order reflections. Panel (c) is an RSM in asymmetric geometry centered on the $\operatorname{GaAs}(422)$ reflection with diffraction features labeled.

we would expect to see only one of these peaks in a given RSM. However, two different epitaxial orientations of $\mathrm{c}-\mathrm{MnSb}$ are present corresponding to growth on $\mathrm{AB}$ versus $\mathrm{AC}$ terminated $\mathrm{n}-\mathrm{MnSb}$ regions. These are mutually misoriented in-plane by $60^{\circ}$. The presence of two epitaxial orientations enhances the grain contrast observed in TEM (Fig. 2). Having both in-plane (4 peaks) and out-ofplane (3 peaks) XRD data for the c-MnSb enables its strain state to be investigated. The lattice parameter is $a=(6.435 \pm 0.007) \AA$ and we cannot detect any significant distortion (fitting three separate lattice parameters always converges to identical values within the experimental error). This value is approximately $1 \%$ smaller than previously reported for c-MnSb in films grown directly on GaAs. ${ }^{5}$ The epitaxial strain for c-MnSb, at this lattice parameter, on $\mathrm{n}-\mathrm{MnSb}$ is $10.2 \%$ and with small grain sizes of tens of nanometer it is clear that substantial epitaxial strains are to be expected. The lack of observed distortion probably reflects the complex structure of the polymorphic layer where the $\mathrm{c}-\mathrm{MnSb}$ grains embedded within a n-MnSb matrix are not subject to simple biaxial stress.

The mosaic spread of the film and substrates were obtained from rocking curves, $Q_{x}$ scans, at the principal XRD peak positions (not shown). The full width at half maximum (FWHM) of the lowest diffraction order rocking curves is as follows: $\mathrm{In}_{0.5} \mathrm{Ga}_{0.5}$ As $0.543^{\circ}$, n-MnSb $0.537^{\circ}$ and c-MnSb $1.210^{\circ}$. Clearly, the mosaic spread of the $\mathrm{n}-\mathrm{MnSb}$ film is dominated by that of the virtual substrate, which in turn is due to the formation of the misfit dislocations at the GaAs/InGaAs interface shown in Fig. 1(a). The c-MnSb gives rise to much broader rocking curves due to mismatch-induced mosaic and the smaller grain size, consistent with the TEM and XRD data.

We show in Fig. 4 typical XRD data for $\mathrm{MnSb}$ films grown directly on GaAs(111). The standard diffractogram contains similar peaks to that of Fig. 3(a) with only the virtual substrate peaks missing. In particular, strong substrate and n-MnSb peaks appear and, despite the very different substrate lattice parameter, $\mathrm{c}-\mathrm{MnSb}$ is also present. The lowest order family of peaks is shown in Fig. 3(b). Some additional features appear compared to the virtual substrate samples. The peak labeled 1, lying between the c-MnSb and $\operatorname{GaAs}(111)$ peaks, is due to GaSb(111). This sometimes occurs at the GaAs/ $\mathrm{n}-\mathrm{MnSb}$ interface due to $\mathrm{Ga}$ droplets resulting from the surface preparation of GaAs. The presence of such alternative III-V compounds at the substratefilm interface which can also include InAs forming during MnAs growth on InP, can make identification of cubic TMP phases ambiguous without care. The peak labeled 2, between $\operatorname{GaAs}(111)$ and $\mathrm{n}-\mathrm{MnSb}(0002)$, is due to $\mathrm{n}-\mathrm{MnSb}(1 \overline{1} 01)$. Small crystallites of this alternative epitaxial orientation can appear in MnSb films on GaAs and have also been observed for low-strain NiSb films on GaAs 


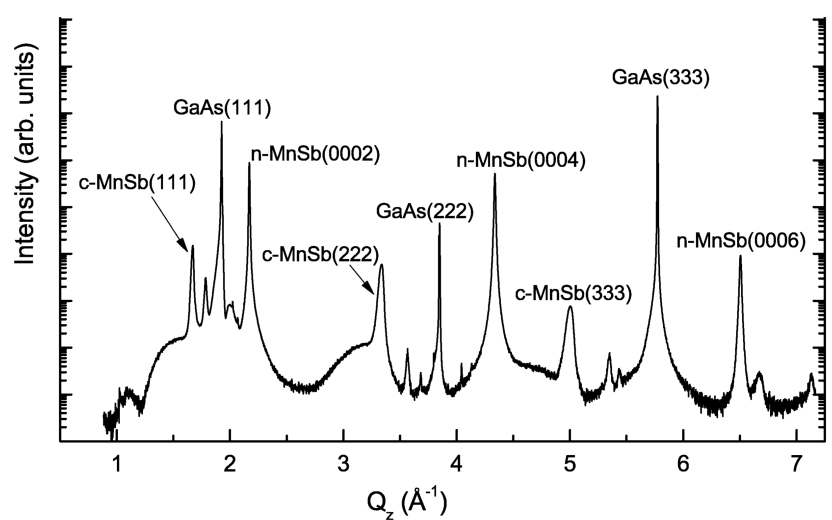

(a)

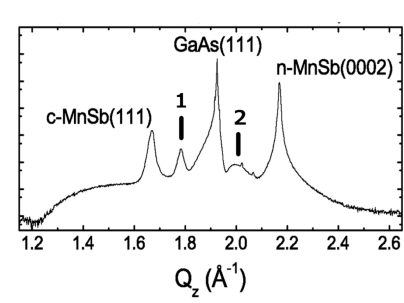

(b)

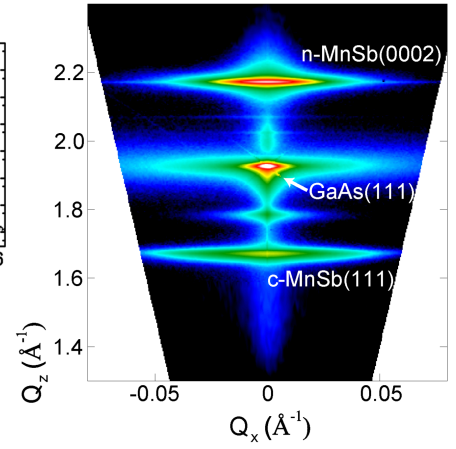

(c)

Fig. 4. Synchrotron XRD data for a typical MnSb/GaAs thin film. Panel (a) shows a standard out-of-plane (symmetric) diffractogram. All the main peaks are labeled and correspond to the expected $\mathrm{GaAs}$ and $\mathrm{In}_{0.5} \mathrm{Ga}_{0.5} \mathrm{As}$ (111)-type reflections and $\mathrm{n}-\mathrm{MnSb}(0002)$ reflections. A clear set of additional weaker peaks is assigned to c-MnSb. Panel (b) shows a more detailed view of the group of lowest order reflections. Panel (c) is an RSM in symmetric geometry showing the lowest order reflections; other features of this RSM are discussed in the text.

(111). ${ }^{22}$ However, the key point is the presence of c-MnSb on both GaAs and $\operatorname{In}_{0.5} \mathrm{Ga}_{0.5}$ As substrates.

A RSM obtained in symmetric diffraction conditions is shown in Fig. 4(c). This data reinforces the interpretation of the principal features of Fig. 4(b). The $\mathrm{n}-\mathrm{MnSb}(0002)$ and GaAs(111) features are intense and symmetric. The peak assigned to GaSb is also symmetric and sharp, reflecting good epitaxy directly on the GaAs substrate; the lattice parameter of $(6.104 \pm 0.003) \AA$ corresponds very well to bulk GaSb (0.13\% expanded). The peak assigned to $\mathrm{n}-\mathrm{MnSb}(1 \overline{1} 01)$ is much weaker and less distinctly symmetrical as expected. The c-MnSb(111) feature is quite broad in $Q_{x}$ as expected for a granular phase with higher mosaic spread; the corresponding rocking curve has FWHM of around $1.1^{\circ}$, similar to its counterpart on $\mathrm{In}_{0.5} \mathrm{Ga}_{0.5}$ As. The other interesting feature of the c-MnSb peak is a broad shoulder extending to lower $Q_{z}$, also very clear in the diffractogram. This can be explained by the presence of very small crystallites in varying high strain states throughout the mixed $\mathrm{n}-\mathrm{MnSb} / \mathrm{c}-\mathrm{MnSb}$ polymorphic layer.

\section{Conclusions and Outlook}

In this work, MnSb thin films have been grown by MBE on (111)-oriented GaAs and $\mathrm{In}_{0.5} \mathrm{Ga}_{0.5} \mathrm{As}$ substrates with lattice mismatches of $3.1 \%$ and $0.31 \%$, respectively. In both cases, high-quality n-MnSb films can be grown using a suitable flux ratio $[6.6: 1$ excess $\mathrm{Sb}]$ and a substrate temperature of $(415 \pm 5){ }^{\circ} \mathrm{C}$. Under these conditions, all films showed the presence of the technologically important c-MnSb polymorph.

Structural characterization by TEM and XRD is consistent with a model of small c-MnSb grains within a mixed polymorphic layer (c- plus n- with sometimes some wurtzite ${ }^{5}$ ). The c-MnSb is epitaxial on and within the n-MnSb matrix, and is under high epitaxial stress. However, this does not appear to be simple biaxial stress due to the mixed nature of the polymorphic layers. There is evidence for a wide range of strain states within the c-MnSb (both among grains in a single film and from film to film) which most likely depends principally on crystallite size. The in-plane epitaxy of the c-MnSb corresponds to (111) planes lying on the $\mathrm{n}-\mathrm{MnSb}(0001)$ but with two possible in-plane orientations separated by $60^{\circ}$, most likely reflecting matching to $\mathrm{AB}$ layer or AC-layer terminated n-MnSb. Further TEM work is under way to understand in more detail the strain states and epitaxial relationship of the c-MnSb in $\mathrm{n}-\mathrm{MnSb}$.

The key point of this paper is that the presence of the c-MnSb polymorphic layers does not depend on whether the $\mathrm{n}-\mathrm{MnSb}$ is sitting on $\mathrm{In}_{0.5} \mathrm{Ga}_{0.5} \mathrm{As}(111)$ or $\operatorname{GaAs}(111)$ substrates. Thus, we can rule out the influence of epitaxial mismatch at the substrate-film interface as the precursor for polymorphic growth, at least in this pair of epitaxial systems. We have never observed c-MnSb grown directly on any III-V or Ge substrate, even for (100) substrates (not discussed here). Hence, we do not believe that $\mathrm{c}-\mathrm{MnSb}$ is readily stabilized directly on semiconductor substrates. At present, the precise mechanism for polymorph nucleation within the $\mathrm{n}-\mathrm{MnSb}$ film remains unknown. It is plausible that the polymorphic 
transition is related to the growth kinetics on particular surface reconstructions of the $\mathrm{n}-\mathrm{MnSb}$ $(0001)^{23}$; layer growth kinetics and nanostructure self-assembly are long known to be affected by surface reconstructions in ordinary III-V semiconductor MBE. ${ }^{24}$ With a better understanding of the TMP polymorph nucleation mechanism, improved precision in the MBE process should allow c-MnSb to be formed more reliably and with tighter control over crystallite sizes. Vicinal substrates may be useful insuppressing the twin epitaxial orientation of the c-MnSb.

The half-metallic cubic TMP polymorphs share key features with the most promising spintronic Heusler alloys, principally high Curie temperature and large minority spin gap. Considering also their good epitaxial compatibility with mainstream semiconductors, these qualities bode well for applications in room-temperature spintronics. Because the c-MnSb crystal structure only contains two facecentered cubic sublattices rather than the four of a full Heusler alloy, there is probably less scope for atomic-scale disorder to disrupt half-metallicity. However, the growth of cubic TMPs is less advanced than that of Heusler alloys and it is not clear what key defect properties may be important in large crystallites. Further DFT calculations of defective cubic TMPs would be valuable. Presently, our experiments on cubic TMPs focus on the crystallite sizes and strain states, which are also issues shared with polycrystalline Heusler alloy films. Experimental demonstration of true half-metallicity in c$\mathrm{MnSb}$ remains challenging due to the co-existence of $\mathrm{n}-\mathrm{MnSb}$ but surface-specific techniques such as spinresolved photoemission may initially be most useful, since surface layers dominated by the cubic polymorph can already be grown. Polymorphic layers thick enough to dominate transport in a point contact Andreev reflection (PCAR) experiment would allow more direct comparison to Heusler alloys for which a significant body of PCAR data exists. The ability to control the strain states of highly spinpolarized cubic TMP layers formed on or within ordinary niccolite-structured layers offers exciting new possibilities in the field of hybrid semiconductor spintronic devices.

\section{Acknowledgments}

This work was supported by EPSRC, UK, under grant numbers EP/I00114X/1 and EP/K032852/1, by the USA's Department of Energy (DE-AC0298CH10886) and by Diamond Light Source. XMaS is a mid-range facility funded by EPSRC. We are grateful to the beamline staff of I16, XMaS and $\mathrm{X} 22 \mathrm{C}$, and to R. Johnston and S. York for expert technical support in Warwick.

\section{References}

1. P. Dowben, J. Phys. Condens. Matter 19, 310301 (2007).

2. R. A. de Groot, F. M. Mueller, P. G. van Engen and K. H. J. Buschow, Phys. Rev. Lett. 50, 2024 (1983).

3. M. I. Katsnelson, V. Y. Irhkin, L. Chioncel, A. I. Lichtenstein and R. A. de Groot, Rev. Mod. Phys. 80, 315 (2008).

4. B. Hillebrands and C. Felser, J. Phys. D: Appl. Phys. 42, 080301 (2009).

5. J. D. Aldous, C. W. Burrows, A. M. Sánchez, R. Beanland, I. Maskery, M. K. Bradley, M. dos Santos Dias, J. B. Staunton and G. R. Bell, Phys. Rev. B 85, 060403(R) (2012).

6. H. Akinaga, T. Manago and M. Shirai, Jpn. J. Appl. Phys. 39, L1118 (2000).

7. J. E. Pask, L. H. Yang, C. Y. Fong, W. E. Pickett and S. Dag, Phys. Rev. B 67, 224420 (2003).

8. M. Ležaić, P. Mavropoulos, J. Enkovaara, G. Bihlmayer and S. Blügel, J. Mater. Sci. 97, 026404 (2006).

9. R. Skomski, J. Appl. Phys. 103, 07 D714 (2008).

10. P. A. Dowben and R. Skomski, J. Appl. Phys. 95, 7543 (2004).

11. T. Graf, S. S. P. Parkin and C. Felser, Prog. Solid State Chem. 39, 1 (2011).

12. J. H. Zhao, F. Matsukura, E. A. K. Takamura, D. Chiba and H. Ohno, Appl. Phys. Lett. 79, 2776 (2001).

13. V. H. Etgens, P. C. deCamargo, M. Eddrief, R. Mattana, J. M. George and Y. Garreau, Phys. Rev. Lett. 92, 167205 (2004).

14. S. J. Hashemifar, P. Kratzer and M. Scheffler, Phys. Rev. B 82, 214417 (2010).

15. H. Oomae, S. Irizawa, Y. Jinbo, H. Toyota, T. Kambayashi and N. Uchitomi, J. Cryst. Growth 378, 410 (2013).

16. H. Oomae, J. T. Asubar, S. Nakamura, Y. Jinbo and N. Uchitomi, J. Cryst. Growth 338, 129 (2012).

17. S. A. Hatfield and G. R. Bell, J. Cryst. Growth 296 , 165 (2006).

18. J. D. Aldous, C. W. Burrows, I. Maskery, M. S. Brewer, T. P. A. Hase, J. A. Duffy, M. R. Lees, C. SánchezHanke, T. Decoster, W. Theis, A. Quesada, A. K. Scmhid and G. R. Bell, J. Phys-Condens. Matter 24, 146002 (2012).

19. C. W. Burrows, A. Dobbie, M. Myronov, T. P. A. Hase, S. B. Wilkins, M. Walker, J. J. Mudd, I. Maskery, 
M. R. Lees, C. F. McConville, D. R. Leadley and G. R. Bell, Cryst. Growth Des. 13, 4923 (2013).

20. P. J. Simmonds, S. N. Holmes, H. E. Beere, I. Farrer, F. Sfigakis, D. A. Ritchie and M. Pepper, J. Vac. Sci. Technol. B 27, 2066 (2009).

21. B. T. M. Willis and H. P. Rooksby, Proc. Phys. Soc. B 67, 290 (1954).

22. J. D. Aldous, C. W. Burrows, I. Maskery, M. Brewer, D. Pickup, M. Walker, J. J. Mudd, T. P. A. Hase,
J. A. Duffy, S. Wilkins, C. Sánchez-Hanke and G. R. Bell, J. Crystal Growth 357, 1 (2012).

23. S. A. Hatfield and G. R. Bell, Surf. Sci. 601, 5368 (2007).

24. B. A. Joyce, D. D. Vvedensky, G. R. Bell, J. G. Belk, M. Itoh and T. S. Jones, Matter. Sci. Eng. B-Solid 67, 7 (1999). 